\title{
Choline-chloride-based eutectic solvent for the efficient production of docosahexaenoyl and eicosapentaenoyl ethanolamides via an enzymatic process
}

\begin{abstract}
Docosahexaenoyl and eicosapentaenoyl ethanolamides (DHEA and EPEA) have physiological functions, including immunomodulation, brain development, and anti-inflammation, but their efficient production is still unresolved. In this study, choline-chloride-based natural deep eutectic solvents are used as media to improve the production of DHEA and EPEA. The water content showed a key effect on the reactant conversion. Adding water to choline chlorideglucose (CG, molar ratio of 5:2) led to a significant increase (13.03\% for EPEA and $27.95 \%$ for DHEA) in the yields after $1 \mathrm{~h}$. The high yields of EPEA (96.84\%) and DHEA $(90.06 \%)$ were obtained under the optimized conditions [fish oil ethyl esters/ethanolamine molar ratio of 1:2, temperature of $60{ }^{\circ} \mathrm{C}, 1 \mathrm{~h}$, enzyme loading of 2195 units, and CG containing $8.50 \%$ water of $43.30 \%$ (w/w, relative to total reactants)]. The products could be easily separated using centrifugation. In summary, the research has the potential to produce fatty acyl ethanolamides.
\end{abstract}

Keyword: Natural deep eutectic solvents; Docosahexaenoyl ethanolamide; Eicosapentaenoyl ethanolamide; Enzymatic synthesis 